\title{
Mappings of finite distortion: Removable singularities
}

\author{
Kai Rajala
}

University of Jyväskylä

Department of Mathematics and Statistics

2003 


\section{Acknowledgements}

I wish to express my gratitude to my advisor Professor Pekka Koskela for his excellent guidance and for teaching me mathematics. Also, I would like to thank the people at the Department of Mathematics and Statistics of the University of Jyväskylä, as well as Professors Juha Heinonen and Nages Shanmugalingam, for their support. I am grateful to Stephen Keith, Jani Onninen and Kevin Rogovin for helpful conversations. Thanks are also due to my parents for their support and encouragement throughout my studies.

I would like to thank the Department of Mathematics of the University of Michigan for hospitality during the academic year 2001-2002. The financial support from the Magnus Ehrnroothin säätiö and the Vilho, Yrjö and Kalle Väisälän rahasto foundations is gratefully acknowledged.

Jyväskylä, October 2003

Kai Rajala 


\section{List of included articles}

This dissertation consists of an introductory part and the following publications:

[A ] Koskela, P., Rajala, K.: Mappings of finite distortion: Removable singularities, Israel J. Math., 136 (2003), 269-283.

[B ] Rajala, K.: Mappings of finite distortion: Removable singularities for locally homeomorphic mappings, Proc. Amer. Math. Soc., to appear.

[C ] Rajala, K.: Mappings of finite distortion: Removability of Cantor sets, Preprint 276, Department of Mathematics and Statistics, University of Jyväskylä, 2003.

In this introductory part these articles will be referred to $[A],[B], .$. , whereas other references will be numbered as [1], [2], ... .

The author of this dissertation has actively taken part in research of the joint paper $[A]$. 


\section{Introduction}

When a particular class of mappings (or functions) in a Euclidean space is studied, the following question often arises: Suppose you are given a domain, i.e. an open and connected set $\Omega \subseteq \mathbb{R}^{n}$, and some small closed set $E \subseteq$ $\Omega$. Consider mappings $f: \Omega \backslash E \rightarrow \mathbb{R}^{n}$ belonging to the class you are studying. Then what kinds of assumptions on $f$ and $E$ guarantee that $f$ can be extended to a mapping defined in entire $\Omega$ and also belonging to the desired class? In mathematical language this question reads as: Under which conditions is the set $E$ removable for mappings $f$ belonging to some particular mapping class? In this work we study the removability question for the class of mappings of finite distortion. We next introduce these mappings and some of their basic properties.

Let $\Omega \subseteq \mathbb{R}^{n}$ be a domain. We call a mapping $f \in W_{\text {loc }}^{1,1}\left(\Omega, \mathbb{R}^{n}\right)$ a mapping of finite distortion if it satisfies

$$
\|D f(x)\|^{n} \leq K(x, f) J(x, f) \quad \text { a.e. }
$$

where $1 \leq K(x, f)<\infty$, and if also $J(\cdot, f) \in L_{\text {loc }}^{1}(\Omega)$. Here $\|D f(x)\|$ stands for the operator norm of the differential matrix of $f$ at a point $x$, while $J(x, f)$ denotes the determinant of $D f(x)$. We shall also consider mappings with values in the compactified Euclidean space $\overline{\mathbb{R}}^{n}$, equipped with the chordal metric defined by

$$
\left\{\begin{array}{l}
q(x, y)=\frac{|x-y|}{\sqrt{\left(1+|x|^{2}\right)\left(1+|y|^{2}\right)}}, \quad x, y \neq \infty \\
q(x, \infty)=\frac{1}{\sqrt{1+|x|^{2}}} .
\end{array}\right.
$$

Then, by conformality of Möbius transformations

$$
g:\left(\overline{\mathbb{R}}^{n} \backslash\{p\}, q\right) \rightarrow\left(\mathbb{R}^{n},|\cdot|\right)
$$

(here $|\cdot|$ stands for the Euclidean norm), we can naturally extend the definition of a mapping of finite distortion to $\overline{\mathbb{R}}^{n}$-valued mappings as follows: Let $f: \Omega \rightarrow \overline{\mathbb{R}}^{n}$ be a mapping, $\Omega \subseteq \mathbb{R}^{n}$. Then $f$ is a mapping of finite distortion, if each $x \in \Omega$ has a neighborhood $U \subseteq \Omega$ so that $g \circ f_{U}: U \rightarrow \mathbb{R}^{n}$ is a mapping of finite distortion for some Möbius transformation $g$, where $f_{U}$ is the restriction of $f$ to $U$.

Mappings of finite distortion have recently been intensively studied. There are two main motivations for the development of this theory. The first one comes from nonlinear elasticity. It has turned out that the mathematical theory of certain physical actions of materials has close connections with the theory of mappings of finite distortion. In fact, the study of such actions 
often leads to problems involving properties of some mapping classes that are similar to those studied in the theory of mappings of finite distortion. See [29] for this type of research.

Secondly, if we require $K \in L^{\infty}(\Omega)$ in the above definition, we then recover the class of quasiregular mappings, also called mappings of bounded distortion. For this class of mappings a rich theory has been developed, starting from Reshetnyak's work in the late 1960s. This theory generalizes classical function theory to higher dimensions in a beautiful way. See the monographs [31], [36] and [38] for the theory of quasiregular mappings. It has recently been noticed that many basic properties of quasiregular mappings also hold for mappings of finite distortion for which some additional conditions are assumed. Furthermore, these assumptions have turned out to be very sharp. A motivation for the development of this theory is to see how well the theory of quasiregular mappings generalizes to this more general situation. This work deals with the latter of the two motivations.

Although special classes of quasiregular mappings had already appeared in some works before, it was Reshetnyak who first gave the definition in its current form, and proved their fundamental properties. In [32], [33] and [34] he proved that non-constant quasiregular mappings are continuous, open and discrete, and that they map sets of measure zero to sets of measure zero. Soon after the works of Reshetnyak, Martio, Rickman and Väisälä came up with three publications ([26], [27] and [28]) studying quasiregular mappings from many different viewpoints. Zorich [40] studied quasiregular local homeomorphisms $f: \mathbb{R}^{n} \rightarrow \mathbb{R}^{n}$, and proved that such mappings are in fact global homeomorphisms when $n \geq 3$. This theorem is called the global homeomorphism theorem. Notice that this theorem fails to be true in the plane, which is shown by the exponential mapping $z \rightarrow \exp (z)$. Martio, Rickman and Väisälä [26], Poletsky [30], and Väisälä [39] established modulus inequalities that are, together with nonlinear partial differential equations used already by Reshetnyak, the most important tool in the theory of quasiregular mappings. By using these inequalities, Rickman ([36] and the references therein) developed a beautiful value distribution theory for quasimeromorphic mappings, i.e. for quasiregular mappings $f: \Omega \rightarrow \overline{\mathbb{R}}^{n}$.

Already in 1976 Gol'dstein and Vodop'yanov [8] proved that mappings of finite distortion belonging to the Sobolev class $W_{\text {loc }}^{1, n}$ are continuous. In 1990s papers showing that Reshetnyak's other above-mentioned results also hold under relaxed assumptions started to appear. In the first results it was shown that the discreteness and openness properties hold true for mappings in the Sobolev class $W_{\text {loc }}^{1, n}$, whose distortion function has some suitable integral bounds, see [16], [9], [25]. In these works the Sobolev regularity assumption was essentially used. It is natural that it is simpler to use In- 
equality (1.1) if it is assumed that the left hand side is locally integrable. Thus it was a novel question to determine if Reshetnyak's results remained valid under weaker Sobolev regularity assumptions.

Perhaps the first work dealing with mappings of finite distortion with milder Sobolev regularity assumptions was David's paper [5], where he proved existence theorems for Beltrami equations with $\|\mu\|_{\infty}=1$ in the plane. Recently in [13], [18] and [19] it was shown that the definition of mappings of finite distortion above, together with the requirement that the distortion function $K(\cdot, f)$ should be locally exponentially integrable to some positive power, implies the fundamental results of Reshetnyak in all dimensions. Furthermore, in [20] exponential integrability of the distortion function was replaced by a more general Orlicz-condition, and theorems and examples were given to show that this condition is sharp in the Orlicz scale for all of the above-mentioned fundamental properties of Reshetnyak. Let us introduce this condition.

Let $\Phi:[0, \infty) \rightarrow[0, \infty)$ be a strictly increasing, differentiable function. We call such a function an Orlicz-function. Now assert the following conditions:

$(\Phi-1) \int_{1}^{\infty} \frac{\Phi^{\prime}(t)}{t} d t=\infty$

$(\Phi-2) t \Phi^{\prime}(t)$ increases to infinity when $t \rightarrow \infty$.

Then the assumption is that for a mapping of finite distortion $f$ there should exist a $\Phi$, satisfying conditions $(\Phi-1)$ and $(\Phi-2)$, such that

$$
\exp (\Phi(K(\cdot, f))) \in L_{l o c}^{1}(\Omega) .
$$

Very recently Koskela and Onninen [22] proved counterparts of the modulus inequalities of quasiregular mappings for mappings of finite distortion satisfying the previous assumption (actually assumptions on the distortion function can be relaxed if more Sobolev regularity is assumed). These inequalities are an important tool in the study of mappings of finite distortion, and they will most likely lead to many counterparts of theorems of quasiregular mappings. As an example we mention that in [23] these inequalities were used to show that Zorich's global homeomorphism theorem mentioned above also holds under Assumption (1.2). In fact, in [23] a more general theorem by Martio, Rickman and Väisälä [28] is generalized. For the analytical theory of mappings of finite distortion, see the monograph [15].

Let us close this section by pointing out that, although it was presented above that mappings of finite distortion satisfying Assumption (1.2) have the same basic properties as quasiregular mappings, they form a larger mapping class. In particular, Assumption (1.2) does not imply quasiregularity. 


\section{Removable singularities for quasiregular map- pings}

In this section we recall some basic removability theorems in the theory of quasiregular mappings. It should be mentioned that, in the planar case, the theory of quasiregular mappings, as well as the theory of mappings of finite distortion, is somewhat different from the higher dimensional theory. The main reason for this is the availability of the Beltrami equation. For example, every mapping $f$ of finite distortion in a plane domain, satisfying Assumption (1.2), admits a factorization as a composition of a homeomorphic mapping of finite distortion $h$ and an analytic function $g$. Moreover, the distortion function of $h$ is majorized by the distortion function of $f$. Thus, in the case when $f$ is a $K$-quasiregular mapping, $h$ is a $K$-quasiconformal homeomorphism. See [5], [16], [2] and [15] for results in dimension two.

Let us introduce one application of the above-mentioned factorization theorem to removable singularities of mappings of finite distortion in the plane. Painlevé's classical theorem says the following: Suppose that $f$ : $\Omega \backslash E \rightarrow \mathbb{C}$ is a bounded analytic function, and that $E \subseteq \Omega$ is a closed set with linear Hausdorff measure zero. Then $f$ can be extended to an analytic function $f: \Omega \rightarrow \mathbb{C}$. By using the factorization theorem and modulus of continuity estimates for mappings of finite distortion, a version of Painlevé's theorem can be proved. In this version, proved in [2], the linear measure condition is replaced by a stronger requirement.

The most classical removability theorem for quasiregular mappings in all dimensions is a theorem by Martio, Rickman and Väisälä [27]: Suppose that $f: \Omega \backslash E \rightarrow \overline{\mathbb{R}}^{n} \backslash F$ is a $K$-quasimeromorphic mapping (a $K$-quasiregular mapping into $\overline{\mathbb{R}}^{n}$ ), $E \subseteq \Omega$ a closed set of zero conformal modulus, and $F \subseteq \overline{\mathbb{R}}^{n}$ a closed set of positive conformal modulus. Then $f$ can be extended to a $K$-quasimeromorphic mapping $\tilde{f}: \Omega \rightarrow \overline{\mathbb{R}}^{n}$. Recall that the conformal modulus of a path family $\Gamma$ is defined by

$$
\begin{aligned}
M(\Gamma)= & \inf \left\{\int_{\mathbb{R}^{n}} \rho^{n}(x) d x: \rho: \mathbb{R}^{n} \rightarrow[0, \infty)\right. \text { is a Borel function such that } \\
& \left.\int_{\gamma} \rho \geq 1 \text { for each locally rectifiable } \gamma \in \Gamma\right\} .
\end{aligned}
$$

A closed set $E$ is said to be a set of zero conformal modulus if $M(\Gamma)=0$, where $\Gamma$ is the family of all non-constant paths whose images intersect $E$. A closed set $E$ is said to be a set of positive conformal modulus if it is not of zero conformal modulus. This theorem can be seen as a generalization of Painlevé's theorem, although it is not a direct analog. An extension of 
this theorem was proven by Koskela and Martio [21]. They showed that a removable set $E$ can be allowed to be slightly larger than of zero conformal modulus, as long as it satisfies a certain geometric condition.

Also in the early stages of the theory, Zorich [41] and Agard and Marden [1] were able to use the methods of Zorich's global homeomorphism theorem in order to show the following: Suppose $f: \Omega \backslash\{x\} \rightarrow \overline{\mathbb{R}}^{n}$ is a locally homeomorphic $K$-quasimeromorphic mapping, $n \geq 3$. Then $f$ can be extended to a locally homeomorphic $K$-quasimeromorphic mapping $\tilde{f}: \Omega \rightarrow \overline{\mathbb{R}}^{n}$. Later, Dairbekov [4] extended this result by showing that the removable set $\{x\}$ can again be replaced by any closed set $E$ of zero conformal modulus. Notice that this theorem fails in the plane, which is shown, for instance, by the analytic function $f: \mathbb{D}(0,1) \backslash\{0\} \rightarrow \mathbb{C} ; f(z)=z^{2}$. One essential feature of this theorem is the fact that nothing has to be assumed from the image set $f(\Omega \backslash E)$.

It was an open problem for a long time whether an analog of Painlevé's theorem holds for quasiregular mappings in higher dimensions, in the strong sense that the removable set is allowed to have positive Hausdorff dimension. This turned out to be true, and was shown first by Iwaniec and Martin [14] in even dimensions, and Iwaniec [11] in all dimensions. For a new proof see [7]. The theorem reads as follows. Let $f: \Omega \backslash E \rightarrow \mathbb{R}^{n}$ be a bounded $K$-quasiregular mapping and $E \subseteq \Omega$ a closed set. Then, if the Hausdorff dimension of $E$ is smaller than a constant depending only on $n$ and $K, f$ can be extended to a $K$-quasiregular mapping $\tilde{f}: \Omega \rightarrow \mathbb{R}^{n}$.

One of the main difficulties in developing the theory of quasiregular mappings has turned out to be the construction of examples proving negative results in higher dimensions. The main contribution for these examples comes from Rickman. One of his constructions, given in [37], is a nonremovability result showing the qualitative sharpness of the previous theorem in dimension three: For each $\lambda>0$ there exists a compact, totally disconnected set $E$ in $\mathbb{R}^{3}$ with Hausdorff dimension smaller than $\lambda$ and a bounded $K(\lambda)$-quasiregular mapping $f: \mathbb{R}^{3} \backslash E \rightarrow \mathbb{R}^{3}$ that does not extend continuously to any point of $E$. Furthermore, the set $E$ can be constructed as a self-similar Cantor set. It is an open question if Rickman's construction can be modified in order to obtain similar examples also in dimensions higher than three. However, such examples are believed to exist.

In the previous removability theorems, one of the essential assumptions was that the image of the mapping should not cover too much of the target space, unless the mapping is a local homeomorphism in dimension at least three. On the other hand, Carleson [3] has proved that there exists a Cantortype set $E$ of the real line, of positive conformal modulus, so that $E$ is removable for meromorphic functions $f: \mathbb{C} \backslash E \rightarrow \overline{\mathbb{C}} \backslash\left\{a_{1}, a_{2}, a_{3}, a_{4}\right\}$. This 
theorem can be seen as kind of a generalization of Picard's theorem on entire meromorphic functions, and it shows that geometrically regular sets can be shown to be removable under assumptions that are essentially milder than in the case of general sets. Järvi and Vuorinen [17] gave an extension of Carleson's result by proving the following: Let $f: B(0,1) \backslash E \rightarrow \overline{\mathbb{R}}^{n}$ be a $K$-quasimeromorphic mapping omitting a finite set $A=\left\{a_{1}, \ldots, a_{p}\right\}$, where $p$ depends only on $K$ and the dimension $n$, and where $E \subseteq B(0,1)$ is a self-similar Cantor set constructed in a certain way. Then there exists a constant $t>0$ depending only on $K, n, p$ and $\inf _{a_{i} \neq a_{j} \in A} q\left(a_{i}, a_{j}\right)$, so that if the Hausdorff dimension of $E$ is smaller than $t$, then $f$ can be extended to a $K$-quasimeromorphic mapping of the unit ball. This theorem strongly relies on the Picard-Schottky -type theorem proved for quasiregular mappings by Rickman [35].

\section{Removable singularities for mappings of fi- nite distortion}

In the next three subsections we discuss removability theorems for mappings of finite distortion. These theorems generalize the previously presented theorems of quasiregular mappings. There are three basic methods that arise in removability questions of quasiregular mappings: Analytical methods that rely on partial differential equations and Sobolev-space techniques, topological methods first used by Zorich, and the use of modulus inequalities. These methods share some common techniques but the role of different methods can be recognized. The main purpose of this work is to show that each of these methods can be applied also in the more general framework of mappings of finite distortion.

\subsection{Analytical methods}

In the theory of mappings of finite distortion, one of the most crucial observations has been the fact that suitable exponential integrability of the distortion function of a mapping of finite distortion $f$ is sufficient for integration by parts against the Jacobian determinant of $f$, see [15]. This means that the formula

$$
\int_{\Omega} \phi J(x, f) d x=-\int_{\Omega} f_{i} J\left(x, f_{1}, \ldots, f_{i-1}, \phi, f_{i+1}, \ldots, f_{n}\right) d x
$$

holds for each $i=1, \ldots, n$ and all $\phi \in C_{0}^{\infty}(\Omega)$. It turns out that the method of integration by parts, combined with Sobolev-space techniques and suitable 
sharp inequalities, leads to strong theorems for mappings of finite distortion. Again, Assumption (1.2) has turned out to be a sharp condition for integration by parts. In [12] such method was used in order to show that closed sets with vanishing $L^{n} \log ^{n-1} L$-capacity are removable for bounded mappings of finite distortion $f$, for which the function $\exp (\lambda K(\cdot, f))$ is locally integrable for some $\lambda>0$. In $[\mathrm{A}]$, Theorem 1.1 we show, by using methods similar to those used in [12], that an analogous theorem holds also if the assumption on the distortion function is changed to Assumption (1.2). Let us state this theorem explicitly.

First define

$$
\psi(t)=t \exp (\Phi(t))
$$

where $\Phi$ is as in Assumption (1.2). Because $\psi$ is strictly increasing, we may define an increasing function $h:[0, \infty) \rightarrow[0, \infty)$ by setting

$$
h(t)=t^{n}\left(\psi^{-1}\left(t^{2 n}\right)\right)^{n-1} .
$$

Now, a compact subset $E \subseteq \Omega$ has zero $h$-capacity, $\operatorname{cap}_{h}(E)=0$, if

$\inf \left\{\int_{\Omega} h(|\nabla u|): u \in C_{0}^{\infty}(\Omega), u(x)=1 \forall x \in G\right.$ for some open $\left.G \supset E\right\}=0$.

Theorem 3.1. Let $\Phi$ and $h$ be as above, such that the Assumptions ( $\Phi-1)$ and $(\Phi-2)$ hold. Let $E \subseteq \Omega$ be a compact set whose h-capacity is zero. If $f: \Omega \backslash E \rightarrow \mathbb{R}^{n}$ is a bounded mapping of finite distortion such that Assumption (1.2) holds, then $f$ extends to a mapping of finite distortion in $\Omega$.

Since sets of zero conformal modulus are of zero Hausdorff dimension, it is clear that the removable sets provided by Theorem 3.1 are also of dimension zero. On the other hand, we show in $[\mathrm{A}]$ that any singleton is of zero $h$ capacity in the setting of Theorem 3.1. By general principles it then follows that one can construct small Cantor sets with $\operatorname{cap}_{h}(E)=0$.

Assumption (1.2) turns out to be sharp also for Theorem 3.1, which is shown by our second theorem, Theorem 1.2 in $[\mathrm{A}]$.

Theorem 3.2. Let $\Phi$ be an Orlicz-function so that

$$
\int_{1}^{\infty} \frac{\Phi^{\prime}(s)}{s} d s<\infty
$$

Let $Q$ be a closed cube in $\mathbb{R}^{n}$, centered at the origin. Then there exists a bounded, continuous mapping $f: Q \backslash\{0\} \rightarrow \mathbb{R}^{n}$ of finite distortion such that

$$
\int_{Q \backslash\{0\}} \exp (\Phi(K(x))) d x<\infty,
$$

but so that $f$ does not extend to a mapping of finite distortion in $Q$. 
To construct the mapping in Theorem 3.2, we use suitably modified copies of one of the constructions in [20], see also [18] and [14]. Although Theorem 3.1 can be seen as a generalization of the theorems by Martio, Rickman and Väisälä [27] or Iwaniec and Martin [14] and Iwaniec [11], it is still not strong enough to be thought of as an analog of either of the two. In the former of the two theorems the mappings need not be bounded; only omittance of a set of positive conformal modulus is assumed. After [A] was finished, the modulus inequalities of mappings of finite distortion were established in [22]. These inequalities can be used to prove the right analog of the Martio-Rickman-Väisälä theorem. This will be discussed in Section 3.3 below. Also, very recently Faraco, Koskela and Zhong [6] gave, assuming $\exp (\lambda K(\cdot, f))$ to be locally integrable for some $\lambda>0$, an improvement that can be seen as an analog of the latter theorem; they showed that closed sets of zero $L^{n} \log ^{n-1-\epsilon}$-capacity are removable for bounded mappings. As demonstrated in [2], Rickman's nonremovability result mentioned above can be used to prove nonremovability results also for bounded mappings of finite distortion. This is done by composing Rickman's mapping with a suitable homeomorphism of finite distortion.

\subsection{Topological methods}

One of the celebrated theorems in the theory of quasiregular mappings has been Zorich's global homeomorphism theorem [40], stating that for $n \geq 3$, every locally homeomorphic quasiregular mapping $f: \mathbb{R}^{n} \rightarrow \mathbb{R}^{n}$ is in fact a global homeomorphism, i.e. a quasiconformal mapping. Martio, Rickman and Väisälä [28] gave a strong generalization of Zorich's theorem by proving that for $n \geq 3$ there exists a constant $r<1$ depending only on $K$ and the dimension so that every locally homeomorphic $K$-quasiregular mapping $f$ : $B(0,1) \rightarrow \mathbb{R}^{n}$ is homeomorphic in the ball $B(0, r)$. In [23] this theorem was generalized to the class of mappings of finite distortion satisfying Assumption (1.2).

By using the ideas of Zorich's proof, Zorich himself [41] and Agard and Marden [42] were able to prove that a single point is removable for locally homeomorphic quasiregular mappings when $n \geq 3$ (actually, in these papers the assumptions are slightly weaker). Twenty years later Dairbekov [4] proved that a stronger assertion holds; not only a point is removable, but one can take any closed set of zero conformal modulus. In [B] Dairbekov's result is generalized to the class of mappings of finite distortion satisfying Assumption (1.2). In this generalization a weighted modulus is used instead of conformal modulus, the reason being that this weighted modulus is used to express the modulus inequalities of mappings of finite distortion. 
For a mapping $f$ of finite distortion, let us define the $K^{n-1}$-modulus $M_{K^{n-1}(\cdot, f)}(\Gamma)$ of a path family $\Gamma$ in $\mathbb{R}^{n}$ by setting

$$
\begin{array}{r}
M_{K^{n-1}(\cdot, f)}(\Gamma)=\inf \left\{\int_{\mathbb{R}^{n}} \rho^{n}(x) K^{n-1}(x, f) d x: \rho: \mathbb{R}^{n} \rightarrow[0, \infty)\right. \text { is a Borel } \\
\text { function such that } \left.\int_{\gamma} \rho \geq 1 \text { for each locally rectifiable } \gamma \in \Gamma\right\} .
\end{array}
$$

Sets of zero or positive $K^{n-1}$-modulus are defined as in the case of conformal modulus. Our removability theorem, Theorem 1.1 in [B], reads as follows.

Theorem 3.3. Assume $n \geq 3$. Let $f: \Omega \backslash E \rightarrow \overline{\mathbb{R}}^{n}$ be a locally homeomorphic mapping of finite distortion, and assume that $E$ is a compact set of zero $K^{n-1}$-modulus. Moreover, assume that (1.2) holds with $\Phi$ satisfying condi-

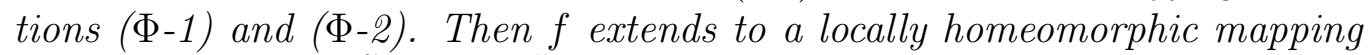
of finite distortion $\tilde{f}: \Omega \rightarrow \overline{\mathbb{R}}^{n}$ satisfying (1.2).

Again, Assumption (1.2) turns out to be sharp. In [20] it is shown that whenever an Orlicz-function $\Phi$ not satisfying Assumption $(\Phi-1)$ is given, there exists a mapping of finite distortion satisfying Assumption (1.2) with $\Phi$, and mapping the punctured unit ball $B(0,1) \backslash\{0\}$ homeomorphically onto an annulus. This mapping certainly does not extend to a local homeomorphism of the unit ball.

Although the modulus inequalities are used in Theorem 3.3, they are only used once in the proof, and that use is of qualitative nature. Also, the modulus inequalities are the only property of quasiregular mappings needed in the proof. Thus most of the proof consists of topological considerations. As mentioned above, these Zorich-type theorems only hold in dimensions higher than of equal to three. The main philosophical reason for this is that, when working in the unit sphere $S^{1}$, one does not have the same topological freedom as in the case of $S^{n}, n \geq 2$. Zorich's global homeomorphism theorem has also had impact in fields other than function theory, see the survey article [42]. Very recently Holopainen and Pankka [10] gave a version of the global homeomorphism theorem for mappings of finite distortion in the setting of Riemannian manifolds.

\subsection{Geometric methods: Modulus inequalities}

As mentioned above, the modulus inequalities of mappings of finite distortion can be used to prove the following analog of the classical theorem of Martio, Rickman and Väisälä. 
Theorem 3.4. If $f: \Omega \backslash E \rightarrow \overline{\mathbb{R}}^{n} \backslash F$ is a mapping of finite distortion satisfying Assumption (1.2), $E \subseteq \Omega$ a closed set of zero $K^{n-1}$-modulus (see the previous section for the definition), and $F \subseteq \overline{\mathbb{R}}^{n}$ a closed set of positive conformal modulus, then $f$ extends to a mapping of finite distortion $\tilde{f}: \Omega \rightarrow$ $\overline{\mathbb{R}}^{n}$ satisfying Assumption (1.2).

To prove this theorem it is essentially sufficient to combine the modulus inequalities and the proof of Martio, Rickman and Väisälä, see [27]. We give the proof in the appendix below, where we also show that actually Theorem 3.4 implies Theorem 3.1.

In this section we also show that the theorem by Järvi and Vuorinen [17], stated in Section 2, admits a generalization for mappings of finite distortion. The proof of the theorem by Järvi and Vuorinen uses the modulus inequalities in a rather quantitative way. There are two main tasks in proving this theorem. First, Rickman's work on the generalization of the Picard-Schottky theorem [35] allows one to prove continuity estimates for quasiregular mappings by only assuming that the mappings in question should omit a large number of points. Secondly, the self-similarity of the removable set, combined with the modulus inequalities, allows one to prove continuity estimates for quasiregular mappings in certain annuli. These annuli converge to each of the exceptional points so that, at the end, one obtains a continuous extension of the mapping to the entire domain, so that the removable set gets mapped to a set of measure zero. Then one is able to use some standard arguments (absolute continuity on almost all lines, topological degree and the area formula) to show that the extension is quasiregular.

In the case of mappings of finite distortion, no results like the PicardSchottky theorem are known. Thus, when generalizing the theorem of Järvi and Vuorinen to the class of mappings of finite distortion, one has to replace the assumption on the omitted set by an essentially stronger one. In $[\mathrm{C}]$ a generalization is given, where the stronger assumption is the omittance of a set of positive conformal modulus, as in the Martio-Rickman-Väisälä theorem. Since the statement of the theorem is quite technical, we only give a simplified version here. This is a special case of Theorem 3.7 in [C].

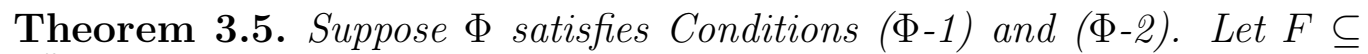
$\overline{\mathbb{R}}^{n}$ be the image of the one-third Cantor set under the stereographic projection. Then there exists a Cantor set $E \subseteq B(0,1)$ so that any mapping of finite distortion $f: B(0,1) \backslash E \rightarrow \overline{\mathbb{R}}^{n} \backslash F$ satisfying

$$
I_{f}=\int_{B(0,1)} \exp (\Phi(K(x, f))) d x<\infty
$$


can be extended to a mapping of finite distortion $f: B(0,1) \rightarrow \overline{\mathbb{R}}^{n}$. The size of the Cantor set in terms of Hausdorff measures with general gauge functions has a lower bound depending only on $\Phi, I_{f}$ and $n$. In the case $\Phi(t)=\lambda t, \lambda>0$, the set $E$ can be chosen so that the Hausdorff measure of $E$ with respect to the gauge function $\Lambda$ is positive, where

$$
\Lambda(t)=|\log t|^{-n \log _{b} 2}, \quad b=\exp \left(C_{n} / \lambda\right) .
$$

In $[\mathrm{C}]$, Theorem 3.7, the omitted set $F$ is only assumed to be of positive conformal modulus. Then the size of the removable set $E$ also depends on the conformal moduli of certain path families related to the set $F$. Since it is assumed in Theorem 3.5 that the omitted set is of positive conformal modulus, this theorem does not give anything new towards a generalization of the Martio-Rickman-Väisälä theorem unless the removable Cantor sets $E$ are of positive $K^{n-1}$-modulus. The last assertion of the theorem implies that there is new insight in this theorem, at least for mappings $f$ whose distortion function $K(\cdot, f)$ has the property $\exp (\lambda K(\cdot, f)) \in L_{\text {loc }}^{1}$ for some $\lambda>0$. For these mappings the removable Cantor sets are actually of positive conformal modulus in the case where $\lambda$ is small enough. It would be interesting to know if one can combine the techniques that are used in proving Theorems 3.3 and 3.5 in order to show that there are sets $E$, of positive conformal modulus (or even larger), so that locally homemorphic mappings of finite distortion $f: \Omega \backslash E \rightarrow \overline{\mathbb{R}}^{n}, n \geq 3$, with integral bounds on the distortion function can be extended to the set $E$ (without assuming anything of the set $f(\Omega \backslash E)$ ). As far as we know, no theorems of this type exist even for quasiregular mappings. 


\section{Appendix: Proof of Theorem 3.4}

We first recall Lemma III 2.6 from [36].

Lemma 3.6. Let $F$ be a compact proper subset of $\overline{\mathbb{R}}^{n}$ and suppose $F$ is of positive conformal modulus. Then for every $a>0$ there exists $\delta>0$ such that $M(\Gamma) \geq \delta$, whenever $C$ is a continuum in $\overline{\mathbb{R}}^{n} \backslash F$ with chordal diameter greater than $a$, where $\Gamma$ is the family of all paths joining $F$ and $C$.

Now suppose $\Omega, f, E$ and $F$ are as in Theorem 3.4. Since $E$ is clearly of zero Hausdorff dimension, the set $\Omega \backslash E$ is a domain. We first show that the mapping $f$ extends continuously to every point $b \in E$. Without loss of generality we may assume that $b=0$. Fix an open ball $B(0, R) \subset \subset \Omega$. Now suppose that $f$ does not have a continuous extension to 0 . Then there exist a constant $a>0$ and sequences $\left(x_{j}\right)$ and $\left(y_{j}\right)$ in $\Omega \backslash E$ such that $r_{j}=\left|x_{j}-y_{j}\right| \rightarrow 0$ and $q\left(f\left(x_{j}\right), f\left(y_{j}\right)\right) \geq a$ for all $j$. Now, for each $j$, one can find a path $\gamma_{j}$ joining $x_{j}$ and $y_{j}$ in $\bar{B}\left(0, r_{j}\right)$. Consider the family $\Gamma_{j}$ of all paths joining the image $\left|\gamma_{j}\right|$ of $\gamma_{j}$ and $S^{n-1}(0, R)$. By [22], Theorem 5.3 and the comment thereafter, $M_{K^{n-1}(\cdot, f)}\left(\Gamma_{j}\right) \rightarrow 0$ when $j$ tends to infinity. On the other hand, Theorem 4.1 of [22] implies that also $M\left(f \Gamma_{j}\right) \rightarrow 0$. This contradicts Lemma 3.6. Thus $f$ has a continuous extension $\tilde{f}: \Omega \rightarrow \overline{\mathbb{R}}^{n}$.

To verify that $\tilde{f}$ is a mapping of finite distortion satisfying Assumption (1.2) it suffices to show that $\tilde{f}$ has locally integrable weak derivatives and that the Jacobian of $\tilde{f}$ is locally integrable. Since the mapping $f$ is absolutely continuous on almost all lines parallel to the coordinate axis (ACL) and the set $E$ is of zero Hausdorff dimension, $\tilde{f}$ is also ACL. Also, since the set $E$ gets mapped to a set of zero Hausdorff dimension and $f$ maps sets of measure zero to sets of measure zero, $|\tilde{f}(A)|=0$ whenever $|A|=0$. We shall prove the local integrability of the Jacobian of $\tilde{f}$ by using the topological index.

Let $x_{0} \in E$. Then $E \cup \tilde{f}^{-1}\left(\tilde{f}\left(x_{0}\right)\right)$ is totally disconnected, since $E$ is of Hausdorff dimension zero and $f$ is discrete. Thus there exists a sphere $S^{n-1}\left(x_{0}, \epsilon\right)$ so that $S_{\tilde{f}}^{n-1}\left(x_{0}, \epsilon\right) \cap \tilde{f}^{-1}\left(\tilde{f}\left(x_{0}\right)\right)=\emptyset$. Let $V$ be the $\tilde{f}\left(x_{0}\right)$ component of $\overline{\mathbb{R}}^{n} \backslash \tilde{f} S^{n-1}\left(x_{0}, \epsilon\right)$ and let $U$ be the $x_{0}$-component of $\tilde{f}^{-1} V$. Then $U$ is an open neighborhood of $x_{0}$, since $U \subseteq B\left(x_{0}, \epsilon\right)$. The topological degree $\mu\left(\tilde{f}\left(x_{0}\right), \tilde{f}, U\right)$ is now well-defined, and we have

$$
N(y, \tilde{f}, U) \leq \mu\left(\tilde{f}\left(x_{0}\right), \tilde{f}, U\right)=m<\infty,
$$

see [26]. Here $N(y, \tilde{f}, U)=\operatorname{card}\{x \in U: \tilde{f}(x)=y\}$. On the other hand by [24], Theorem 9.2, the area formula holds for $\tilde{f}$ in $U \backslash E$, since $\tilde{f} \in$ $W_{\text {loc }}^{1,1}\left(U \backslash E, \mathbb{R}^{n}\right)$ and the Lusin condition holds for $\tilde{f}$. Thus

$$
\int_{U} J(x, \tilde{f})=\int_{U \backslash E} J(x, \tilde{f})=\int_{\mathbb{R}^{n}} N(y, \tilde{f}, U \backslash E) \leq|V| \mu\left(\tilde{f}\left(x_{0}\right), \tilde{f}, U\right)<\infty .
$$


We conclude that $J(x, \tilde{f})$ is locally integrable. Since $\tilde{f}$ is ACL and has locally integrable Jacobian, the distortion inequality (1.1), exponential integrability of the distortion function and Hölder's inequality together imply that $\tilde{f} \in$ $W_{\text {loc }}^{1,1}\left(\Omega, \mathbb{R}^{n}\right)$. The proof is complete.

Let us close by showing that Theorem 3.4 implies Theorem 3.1. For this we need to show that the $K^{n-1}$-modulus of a compact set $E$ is zero whenever the $h$-capacity of $E$ is zero. We first prove that the vanishing of the $h$ capacity of a compact set $E$ does not depend on the open set $U$ in which the supports of the test functions are assumed to lie, as long as $E \subseteq U$. Recall the definitions of the functions $\psi$ and $h$ from (3.1) and (3.2), respectively.

Lemma 3.7. Let $E$ be a compact subset of a bounded open set $U$. Suppose that $E$ is of zero h-capacity with respect to $U$. Let $V$ be another bounded open set containing $E$. Then $E$ is also of zero h-capacity with respect to $V$.

Proof. Let $u_{i}$ and $v$ be test functions for the $h$-capacity, so that $u_{i} \in C_{0}^{\infty}(U)$, $v \in C_{0}^{\infty}(V)$ and $\int h\left(\left|\nabla u_{i}\right|\right)<1 / i$. We may assume that $0 \leq v, u_{i} \leq 1$ and that $u_{i} \in C_{0}^{\infty}\left(\mathbb{R}^{n}\right)$. Now the function $u_{i} v \in C_{0}^{\infty}(V)$ is a test function for the $h$-capacity. Note that $\left|\nabla\left(u_{i} v\right)\right| \leq u_{i}|\nabla v|+v\left|\nabla u_{i}\right|$, and thus

$$
\int_{V} h\left(\left|\nabla\left(u_{i} v\right)\right|\right) d x \leq \int_{V} h\left(u_{i}|\nabla v|+v\left|\nabla u_{i}\right|\right) d x \leq \int_{V} h\left(M u_{i}+\left|\nabla u_{i}\right|\right) d x
$$

where $M=\max |\nabla v(x)|$. From the proof of $[\mathrm{A}]$, Lemma 2.5 we have the estimate $h^{\prime}(t) \leq t^{n}+C$, where $C$ depends only on $n$ and $\Phi$. Hence we can further estimate the last term in order to have

$$
\begin{aligned}
\int_{V} h\left(M u_{i}+\left|\nabla u_{i}\right|\right) d x & =\int_{V} h\left(\left|\nabla u_{i}\right|\right) d x+\int_{V} \int_{\left|\nabla u_{i}\right|}^{\left|\nabla u_{i}\right|+M u_{i}} h^{\prime}(t) d t d x \\
& \leq \int_{V} h\left(\left|\nabla u_{i}\right|\right) d x+\int_{V} \int_{\left|\nabla u_{i}\right|}^{\left|\nabla u_{i}\right|+M u_{i}} t^{n}+C d t d x
\end{aligned}
$$

The first term is bounded by $1 / i$, while the second term is smaller than

$$
C \int_{V} u_{i}+\left|\nabla u_{i}\right|^{n}+\left|\nabla u_{i}\right|^{n-1}+\ldots+\left|\nabla u_{i}\right|+u_{i}^{n+1} d x
$$

where $C$ depends only on $n, \Phi$ and $M$. By the Sobolev-Poincaré inequality we have an exponent $p<n$ so that

$$
\int_{V} u_{i}^{n+1} d x \leq C(n)\left(\int_{V}\left|\nabla u_{i}\right|^{p} d x\right)^{(n+1) / p} .
$$


Moreover, by Hölder's inequality we have

$$
\int_{V} u_{i} d x \leq C(n,|V|)\left(\int_{V}\left|\nabla u_{i}\right|^{p} d x\right)^{1 / p} .
$$

By Hölder's inequality we can further estimate all terms in (3.4) by corresponding terms that are of the form

$$
C(n, \Phi, M,|V|)\left(\int_{V}\left|\nabla u_{i}\right|^{n} d x\right)^{r} .
$$

Note that neither the number of these terms nor the values of the constants depend on $i$. We now choose

$$
A=\left(\psi\left(i^{\frac{1}{2(1-n)}}\right)\right)^{\frac{1}{2 n}}
$$

and estimate (recall that $\left.h(t)=t^{n}\left(\psi^{-1}\left(t^{2 n}\right)\right)^{n-1}\right)$

$$
\begin{aligned}
\int_{V}\left|\nabla u_{i}\right|^{n} d x & =\int_{\left\{\left|\nabla u_{i}\right| \leq A\right\}}\left|\nabla u_{i}\right|^{n} d x+\int_{\left\{\left|\nabla u_{i}\right|>A\right\}}\left|\nabla u_{i}\right|^{n} d x \leq|V| A^{n} \\
& +\int_{\left\{\left|\nabla u_{i}\right|>A\right\}} h\left(\left|\nabla u_{i}\right|\right)\left(\psi^{-1}\left(|\nabla u|^{2 n}\right)\right)^{1-n} d x \leq|V| A^{n}+i^{-1 / 2} .
\end{aligned}
$$

Combining all the estimates, we have that

$$
\int_{V} h\left(\left|\nabla\left(u_{i} v\right)\right|\right) d x \leq T(i)
$$

where $T(i) \rightarrow 0$ as $i \rightarrow \infty$. The proof is complete.

Now let $E$ be a compact set of $h$-capacity zero, contained in an open set $U$. Moreover, let $u \in C_{0}^{\infty}(U)$ with $u=1$ in some neighborhood of $E$. Clearly

$$
\int_{\gamma}|\nabla u| d s \geq 1
$$

for all locally rectifiable curves $\gamma$ joining $E$ and the complement of $U$. We want to estimate the integral $\int_{U}|\nabla u(x)|^{n} K^{n-1}(x) d x$. We consider two cases. First, in the set

$$
U_{1}=\left\{x \in U: K(x) \leq \psi^{-1}\left(|\nabla u(x)|^{2 n}\right)\right\}
$$

we have

$$
\int_{U_{1}}|\nabla u(x)|^{n} K^{n-1}(x) d x \leq \int_{U_{1}} h(|\nabla u(x)|) d x
$$


by the definition of $h$. On the other hand, for $x \in U_{2}=U \backslash U_{1}$, the inequality

$$
|\nabla u(x)|^{n} \leq K^{1 / 2}(x) \exp (\Phi(K(x)))^{1 / 2}
$$

holds by the definition of $\psi$, and so

$$
\begin{aligned}
\int_{U_{2}}|\nabla u(x)|^{n} K^{n-1}(x) d x & \leq \int_{U_{2}} K^{n-1 / 2}(x) \exp (\Phi(K(x)))^{1 / 2} d x \\
& \leq C(\Phi) \int_{U_{2}} \exp (\Phi(K(x))) d x
\end{aligned}
$$

Here we need the assumption that $\Phi^{\prime}(t) t \rightarrow \infty$ as $t \rightarrow \infty$. By combining the estimates on integrals over $U_{1}$ and $U_{2}$, we arrive at

$$
\int_{U}|\nabla u(x)|^{n} K^{n-1}(x) d x \leq C \int_{U} \exp (\Phi(K(x)))+h(|\nabla u(x)|) d x .
$$

Recall that the function $x \rightarrow \exp (\Phi(K(x)))$ is assumed to be locally integrable. Hence we can, for arbitrary $\epsilon>0$, choose open sets $V_{i}, i \in \mathbb{N}$ so that $E \subseteq V_{i}$ and

$$
\int_{V_{i}} \exp (\Phi(K(x))) d x \leq \epsilon 2^{-i}
$$

for all $i \in \mathbb{N}$. Furthermore, since the $h$-capacity of $E$ is zero we find, by Lemma 3.7, smooth functions $u_{i}$, supported in $V_{i}$, so that $u_{i}=1$ on a neighborhood of $E$ and

$$
\int_{V_{i}} h\left(\left|\nabla u_{i}(x)\right|\right) d x \leq \epsilon 2^{-i} .
$$

Applying (3.6), (3.7) and (3.8), we conclude that

$$
\int_{V_{i}}\left|\nabla u_{i}(x)\right|^{n} K^{n-1}(x) d x \leq 2^{-i+1} \epsilon .
$$

Set

$$
\rho(x)=\max _{i \in \mathbb{N}}\left|\nabla u_{i}(x)\right| .
$$

Then $\rho$ is a Borel function and (3.5), with $|\nabla u|$ replaced by $\rho$, holds for all locally rectifiable curves intersecting $E$. By (3.9),

$$
\int_{\mathbb{R}^{n}} \rho^{n}(x) K^{n-1}(x) d x \leq \sum_{i=1}^{\infty} \int_{V_{i}}\left|\nabla u_{i}(x)\right|^{n} K^{n-1}(x) d x \leq 2 \epsilon .
$$

Hence $E$ is of zero $K^{n-1}$-modulus. 


\section{References}

[1] Agard, S., Marden, A.: A removable singularity theorem for local homeomorphisms, Indiana Univ. Math. J., 20 (1970), 455-461.

[2] Astala, K. Iwaniec, T. Koskela, P., Martin, G.: Mappings of BMObounded distortion, Math. Ann. 317 (2000), no. 4, 703-726.

[3] Carleson, L.: A remark on Picard's theorem, Bull. Amer. Math. Soc., 67 (1961), 142-144.

[4] Dairbekov, N. S.: Removable singularities of locally quasiconformal mappings, Siberian Math. J., 33 (1992), no. 1, 159-161.

[5] David, G.: Solutions de l'equation de Beltrami avec $\|\mu\|_{\infty}=1$, (French), Ann. Acad. Sci. Fenn. Ser. A 1 Math., 13 (1988), no. 1. 25-70.

[6] Faraco, D., Koskela, P., Zhong, X.: Mappings of finite distortion: The degree of regularity, Preprint Nr. 45/2003, Max Planck Institute for Mathematics in the Sciences.

[7] Faraco, D., Zhong, X.: A short proof of the self-improving regularity of quasiregular mappings, Preprint Nr. 106/2002, Max Planck Institute for Mathematics in the Sciences.

[8] Gol'dstein, V., Vodop'yanov, S.: Quasiconformal mappings and spaces of functions with generalized first derivatives, Sibirsk. Mat. Z., 17 (1976), $515-531$.

[9] Heinonen, J., Koskela, P.: Sobolev mappings with integrable dilatations, Arch. Rational Mech. Anal., 125 (1993), no. 1, 81-97.

[10] Holopainen, I., Pankka, P.: Mappings of finite distortion: Global homeomorphism theorem, Preprint 339, Department of Mathematics, University of Helsinki, 2002.

[11] Iwaniec, T.: p-harmonic tensors and quasiregular mappings, Ann. of Math. (2), 136 (1992), no. 3, 589-624.

[12] Iwaniec, T., Koskela P., Martin, G., Sbordone, C.: Mappings of finite distortion: $L^{n} \log ^{\alpha}$ L-integrability, J. London Math. Soc. (2), 67 (2003), no. $1,123-136$.

[13] Iwaniec, T., Koskela P., Onninen, J.: Mappings of finite distortion: Monotonicity and continuity, Invent. Math. 44 (2001), no. 3, 507-531. 
[14] Iwaniec, T., Martin, G.: Quasiregular mappings in even dimensions, Acta Math. 170 (1993), no. 1, 29-81.

[15] Iwaniec, T., Martin, G. J.: Geometric Function Theory and Nonlinear Analysis, Oxford Mathematical Monographs, 2001.

[16] Iwaniec, T., Šveràk, V.: On mappings with integrable dilatation, Proc. Amer. Math. Soc., 118 (1993), 181-188.

[17] Järvi, P., Vuorinen, M.: Self-similar Cantor sets and quasiregular mappings, J. Reine Angew. Math., 424 (1992), 31-45.

[18] Kauhanen, J., Koskela, P., Malý, J.: Mappings of finite distortion: Discreteness and openness, Arch. Rational Mech. Anal., 160 (2001), 135151.

[19] Kauhanen, J., Koskela, P., Malý, J.: Mappings of finite distortion: Condition N, Michigan Math. J., 9 (2001), no. 1, 169-181.

[20] Kauhanen, J., Koskela, P., Malý, J., Onninen, J., Zhong, X.: Mappings of finite distortion: Sharp Orlicz-conditions, Rev. Mat. Iberoamericana, to appear.

[21] Koskela, P., Martio, O.: Removability theorems for quasiregular mappings, Ann. Acad. Sci. Fenn. Ser. A 1 Math., 15 (1990), no. 2, 381-399.

[22] Koskela, P., Onninen, J.: Mappings of finite distortion: Capacity and modulus inequalities, Preprint 257, Department of Mathematics and Statistics, University of Jyväskylä, 2002.

[23] Koskela, P., Onninen, J., Rajala, K.: Mappings of finite distortion: Injectivity radius of a local homeomorphism, Preprint 266, Department of Mathematics and Statistics, University of Jyväskylä, 2002.

[24] Malý, J.: Lectures on change of variables in integral, Preprint 305, Department of Mathematics, University of Helsinki, 2001.

[25] Manfredi, J., Villamor, E.: Mappings with integrable dilatation in higher dimensions, Bull. Amer. Math. Soc. (N.S.), 32 (1995), no. 2, 235-240.

[26] Martio, O., Rickman, S., Väisälä, J.: Definitions for quasiregular mappings, Ann. Acad. Sci. Fenn. Ser. A I Math., 448 (1969), 1-40.

[27] Martio, O., Rickman, S., Väisälä, J.: Distortion and singularities of quasiregular mappings, Ann. Acad. Sci. Fenn. Ser. A I Math., 465 (1970), $1-13$. 
[28] Martio, O., Rickman, S., Väisälä, J.: Topological and metric properties of quasiregular mappings, Ann. Acad. Sci. Fenn. Ser. A I Math., 488 (1971), 1-31.

[29] Müller, S., Spector, S.: An existence theory for nonlinear elasticity that allows for cavitation, Arch. Rational Mech. Anal., 131 (1995), no. 1, $1-66$.

[30] Poletsky, E. A.: The modulus method for non-homeomorphic quasiconformal mappings, (Russian), Mat. Sb., 92 (1970), 261-272.

[31] Reshetnyak, Yu. G.: Space Mappings with Bounded Distortion, Trans. of Mathematical Monographs, Amer. Math. Soc, vol. 73, 1989.

[32] Reshetnyak, Yu. G.: Bounds on moduli of continuity for certain mappings, (Russian), Sibirsk. Mat. Z., 7 (1966), 1106-1114.

[33] Reshetnyak, Yu. G.: Space mappings with bounded distortion, (Russian), Sibirsk. Mat. Z., 8 (1967), 629-659.

[34] Reshetnyak, Yu. G.: On the condition of the boundedness of index for mappings with bounded distortion, (Russian), Sibirsk. Mat. Z., 9 (1968), 368-374.

[35] Rickman, S.: Quasiregular mappings and metrics on the n-sphere with punctures, Comment. Math. Helv., 59 (1984), no. 1, 136-148.

[36] Rickman, S.: Quasiregular mappings, Ergebnisse der Mathematik und ihrer Grenzgebiete (3) [Results in Mathematics and Related Areas (3)], 26. Springer-Verlag, Berlin, 1993.

[37] Rickman, S.: Nonremovable Cantor sets for bounded quasiregular mappings, Ann. Acad. Sci. Fenn. Ser. A 1 Math., 20 (1995), 155-165.

[38] Vuorinen, M.: Conformal geometry and quasiregular mappings, Lecture Notes in Mathematics, 1319. Springer-Verlag, Berlin, 1988.

[39] Väisälä, J.: Modulus and capacity inequalities for quasiregular mappings, Ann. Acad. Sci. Fenn. Ser. A 1 Math., 509 (1972), 1-14.

[40] Zorich, V. A.: The theorem of M. A. Lavrent'ev on quasiconformal mappings in space, (Russian), Mat. Sb. 74 (1967), 417-433.

[41] Zorich, V. A.: Isolated singularity of mappings with bounded distortion, Mat. Sb., 81 (1970), no. 4, 581-583. 
[42] Zorich, V. A.: Quasi-conformal maps and the asymptotic geometry of manifolds, Russian Math. Surveys, 57 (2002), no. 3, 437-462. 